\title{
Museu de Zoologia da Universidade de São Paulo: adaptação aos novos tempos
}

\author{
MARIA ISABEL LANDIM
}

\section{Introdução}

$\mathrm{O}$ ACERVO do Museu de Zoologia da USP tem origem centenária: soma do acervo eclético do museu particular de Joaquim Sertório com uma coleção menor de um certo senhor Pessanha, mais o acervo do Museu Provincial montado e inaugurado em 1877 pela Sociedade Auxiliadora do Progresso da Província de São Paulo. Essas coleções reunidas formaram o núcleo do que, em 1891, em São Paulo, se chamou Museu do Estado.

Após adquirir o acervo do Museu Sertório, o conselheiro Francisco de Paula Mayrink doou-o ao Estado de São Paulo que o incorporou ao Museu Provincial. O Museu do Estado funcionou de 1891 a 1893 sob a direção do sueco Alberto Löefgren, que já havia trabalhado para Joaquim Sertório, ajudando-o na organização de seu museu. Em 1893, essa coleção com uma expressiva representatividade das diversas áreas da História Natural foi posta sob a responsabilidade da Comissão Geográfica e Geológica do Estado de São Paulo e passou a ser coordenada por Orville Adelbert Derby com o nome de Museu Paulista e em 1894, já desligada da Comissão, passa a ocupar o edifício monumento à independência no Ipiranga. Considerando apenas o final do século XIX, esse acervo esteve reunido sob diferentes instituições prenunciando uma história de reviravoltas institucionais que marcaram essas coleções.

A criação de museus no século XIX no Brasil seguiu a tendência de expansão para fora do eixo Europa-Estados Unidos e esteve associada ao movimento europeu de criação de museus coloniais e exploração da fauna e flora de alémmar e de descentralização da produção de conhecimento. Os museus criados "fora do eixo" mantiveram fortes vínculos com a Europa e Estados Unidos, o que refletiu até na escolha de seus diretores e na presença de grande número de estrangeiros em seus quadros funcionais (Sheets-Pyenson, 1988).

Em sua origem, os museus brasileiros, mesmo que abrigando coleções ecléticas, estavam voltados para o conhecimento dos diversos ramos da história natural brasileira, e a direção de um zoólogo (de 1893 a 1916) no Museu Paulista deu um cunho eminentemente naturalista para a instituição. O que se viu em seguida foi a especialização dos ramos de conhecimento estimulada também 
pela criação dos museus e, consequentemente, pelo aumento de suas coleções. A história natural, porém, que fora impulsionadora do projeto de institucionalização da produção de conhecimento em nosso país, não recebeu a mesma atenção de dirigentes durante os diversos períodos que nos trouxeram aos dias atuais.

\section{Museu Paulista (de História Natural)}

A origem do Museu Paulista, um dos primeiros museus brasileiros, está vinculada à criação do Museu Real (depois Museu Nacional do Rio de Janeiro) em 1818, que previa a criação de uma rede de museus provinciais que estariam conectados ao Museu Metropolitano com uma missão e estratégia relacionadas à propagação das Ciências Naturais no Brasil. Nessa perspectiva, surgiram também importantes museus em nosso país, como o Museu Paraense (Emílio Goeldi) em 1871, em Belém, o Museu Paranaense em 1876, em Curitiba, e o Museu Botânico do Amazonas, em 1883. O vínculo com o Museu Nacional se deu ainda pela designação do primeiro diretor do Museu Paulista, o zoólogo alemão Herman von Ihering, que havia atuado como naturalista viajante do Museu Nacional (Lopes, 1997).

Em São Paulo, o Museu Paulista, que se origina de coleções que tinham o intuito de documentar a vida selvagem do entorno da cidade, como o Museu Sertório (Brandão \& Samara, 2005), dará importante contribuição para a consolidação da pesquisa em diferentes áreas do conhecimento no próspero Estado de São Paulo do final do século XIX e início do XX.

Herman von Ihering estava comprometido com a visão em voga da especialização nos diversos campos das ciências. Chegou a defender a ideia da criação de um museu só de moluscos (seu grupo de estudo), defendendo que esse era o caminho para aprofundar o conhecimento nas diversas áreas. De fato, sem compromisso direto com a docência, a institucionalização da pesquisa em nosso país, nos museus, oferecia a oportunidade e o compromisso de dedicação exclusiva (Lopes \& Figueirôa, 2002-2003) por parte de seus funcionários que se viram envolvidos nas diversas etapas do processo curatorial (sensu Brandão \& Landim, no prelo). Assistimos gradualmente à formação de profissionais locais que continuariam o processo de institucionalização da pesquisa e curadoria científica em nosso país.

Ihering se mostrou conectado com a rede de museus e de produção de conhecimento internacional. Sua preocupação com a produção e disseminação de conhecimento resultou na publicação da Revista do Museu Paulista para dar vazão ao novo campo que se institucionalizava. Dessa forma, ele colocava o $\mathrm{Mu}-$ seu Paulista na rede internacional de intercâmbio de conhecimento e material, buscando sempre estar atualizado nas suas práticas científicas e museológicas.

A separação do acervo do Museu Paulista só começou após a gestão de Ihering. $\mathrm{O}$ processo de especialização pelo qual passou a coleção eclética do Museu Paulista sob a direção do historiador Affonso d'Éscrangnolle Taunay (de 1917 a 1925) ilustra um período de declínio do status da História Natural 
na instituição paulista. Em outros países, por razões diversas, isso também foi observado. Em São Paulo, as diversas coleções do Museu Paulista referentes a diferentes ramos da História Natural (botânica, mineralogia, zoologia, arqueologia e etnologia) seguiram caminhos próprios em instituições mais focadas como o Instituto Biológico, o Instituto de Geociências, o Museu de Zoologia e o Museu de Arqueologia e Etnologia, os três últimos hoje sob a chancela da Universidade de São Paulo. Essa divisão explica em parte a inexistência de um Museu de História Natural atualmente em São Paulo.

Em Londres, em meados do século XIX, em pleno processo de profissionalização da pesquisa em História Natural, as coleções de História Natural do Museu Britânico ficaram enormes e destoantes do resto de seu acervo. Por essa razão, o anatomista Richard Owen, chefe do Departamento de História Natural do museu, empreendeu uma campanha para a criação de novo prédio que abrigasse essas coleções. O novo prédio, no bairro de South Kensington, foi inaugurado em 1881 (Stearn, 1998), com o nome de Museu Britânico (História Natural). Assim, surgia em Londres um “templo” para a História Natural (com um certo grau de interdisciplinaridade) em que coexistiam um projeto arquitetônico conservador e uma nova abordagem museográfica.

Ao contrário do que havia idealizado o fundador do Museu Britânico (História Natural), Richard Owen, um novo modelo de exposição seguindo a nova tendência da época era posto em prática. O plano original de Owen para a sua "catedral" era expor todo e cada exemplar das coleções do museu, para dar a exata dimensão do poderio do Império Britânico (Sheets-Pyenson, 1988, p.7; Stearn, 1998, p.75; Yanni, 2005, p.111). Seus opositores, focados na crescente profissionalização entre os naturalistas, defendiam que apenas alguns animais, de interesse do público, deveriam ser expostos. Dessa forma, a maior parte das coleções era mantida em reservas técnicas. Essas coleções “científicas” estavam à disposição para estudo de pesquisadores. Essa política curatorial, de divisão das coleções em científicas e didáticas ou museográficas, era defendida também pelo zoólogo George Brown Goode, diretor do Museu Nacional dos Estados Unidos, da Smithsoninan Institution (Alves, 2001, p.82). Ihering e Goode eram correspondentes e essa abordagem, hoje paradigmática, também foi aplicada por aqui.

Atualmente, seguindo essa tradição, mesmo que as exposições façam uso de material científico das coleções dos museus, não é raro o uso de réplicas e modelos em suas galerias. As peças raras, frágeis, ou de grande valor científico são preservadas longe do olhar do público. Os objetos expostos devem articular narrativas em exposições que não mais funcionam como álbuns de figurinhas de um microcosmo a ser reconstituído. Essa tensão entre preservar o raro e a informação para o futuro e compartilhá-los com o público ilustra o paradoxo institucional que é característico dos museus (Brandão \& Landim, no prelo). 
O caso específico do Museu de Zoologia da Universidade de São Paulo é um exemplo vívido do processo dinâmico de desenvolvimento da pesquisa em biodiversidade no Brasil. Seguindo o modelo do Museu Nacional, Ihering contratou muitos estrangeiros como naturalistas para realizarem expedições de coletas visando à ampliação das coleções (Menezes et al., 1997). Sob a sua direção, as coleções biológicas do Museu Paulista cresceram de forma desproporcional aos demais acervos. Ainda hoje, os registros mais antigos identificáveis dos exemplares de nossas coleções remetem a esse período, responsável pelo primeiro significativo esforço sistemático de coleta de material. A maior parte das galerias do Museu Paulista expunha a História Natural (Lopes, 1997). Apenas mais tarde, com a nomeação de Affonso d'Éscrangnolle Taunay como diretor, o foco institucional se volta para a vocação do edifício monumento à independência do Brasil e seu acervo histórico.

Nesse processo, criaram-se dentro do Museu Paulista, em 1925, uma Seção de Zoologia e, em 1939, um Departamento de Zoologia ligado à Secretaria de Agricultura, Indústria e Comércio de São Paulo (Figura 1). O atual prédio do Museu de Zoologia começou a ser construído em 1939 para abrigar a extensa coleção zoológica do Museu Paulista. O compromisso com a pesquisa e com a instrução pública a partir do acervo, presente no decreto de criação do Museu Paulista, foi mantido durante todo esse período. Com um projeto de Cristiano Stokler das Neves, foi construído o primeiro prédio em São Paulo com a finalidade de abrigar um museu: um museu de zoologia (Silva, 2006; Neves Neto, 2008, p.87). Em 1941, com o prédio concluído, as coleções zoológicas foram transferidas. Mesmo que já para a época o projeto fosse um pouco subdimensionado para as coleções e pesquisa em zoologia do então Departamento de Zoologia, esse prédio abriga ainda hoje, com todas as suas limitações, uma das maiores e mais importantes coleções da fauna neotropical do mundo (Figura 1).

\section{Museu Universitário (de Zoologia)}

A relação de aproximação do museu com a Universidade de São Paulo, criada em 1934, é antiga, e a ideia de incorporar um museu de história natural à Faculdade de Filosofia Ciências e Letras já aparece em 1936 (Silva, 2006) no relatório anual de atividades de Taunay. O próprio Museu Paulista já era considerado uma unidade complementar à USP desde 1934 (Decreto n.6.283 - criação da USP - de 25 de janeiro de 1934, art. $4^{\circ}$ ). Sob a direção de Olivério Mário de Oliveira Pinto, a partir de 1939, essa ideia se fez recorrente. Mas apenas em 1969, sob a direção de Paulo Emílio Vanzolini, o Museu de Zoologia foi incorporado à Universidade.

Como os demais Institutos de Pesquisa que surgiram na época, o foco do Departamento de Zoologia estava na pesquisa. Contemplava ainda a instrução pública mediante uma exposição que seguia os modelos clássicos de classificação taxonômica dos exemplares exibidos segundo a relação de parentesco entre eles, desde Ihering, contemplando as influências de Carl Linnaeus e Charles Darwin às ciências biológicas. 
Durante a direção de Paulo Emílio Vanzolini (de 1962 a 1992), o Museu de Zoologia se inseriu definitivamente no cenário internacional, e a pesquisa nessa instituição esteve atualizada com as novas metodologias então em voga. Além disso, a inserção na Universidade possibilitou aos pesquisadoresdocentes do MZUSP o engajamento em cursos de pós-graduação e no ensino formal de uma nova geração de sistematas e taxonomistas que começou a ser formada por meio pesquisa das coleções do Museu. A separação da zoologia do Museu Paulista também resultou na publicação dos periódicos Arquivos de Zoologia do Estado de São Paulo (hoje Arquivos de Zoologia), em 1940, e Papéis Avulsos do Departamento de Zoologia, da Secretaria de Agricultura de São Paulo (hoje Papéis Avulsos de Zoologia), em 1941. O foco institucional na pesquisa garantiu ao MZUSP tornar-se referência na produção de conhecimento em biodiversidade. Além disso, assegurou o aumento exponencial de suas coleções, coligidas por meio de grandes projetos de pesquisa em áreas estratégicas do território brasileiro e fora desse. Esforços coletivos dentro da instituição foram realizados (como na Expedição Permanente à Amazônia, financiada pela Fapesp) e resultaram no aumento significativo do acervo do MZUSP. Com o olhar para fora, a pesquisa do Museu de Zoologia se projetou no século XXI. Toda essa expansão de atividade científica se fez mesmo diante do grande declínio no número de docentes-pesquisadores que, em 2001, chegou ao seu limite mínimo de oito docentes, contra os 24 da extinta Seção de Zoologia do Museu Paulista.

Como herdeiro de Herman von Ihering, o MZUSP realizou a promessa de se firmar como instituição de pesquisa, constituindo uma das coleções de fauna Neotropical mais importantes do mundo. ${ }^{1}$ Ao contrário dele, enfrentamos uma urgência na geração de informação sobre a diversidade biológica de nosso país megadiverso ${ }^{2}$ ante o desenvolvimento econômico acelerado e a consequente perda de paisagens naturais e espécies. A extinção atual de espécies provocada pela ação do ser humano no planeta alcança taxas alarmantes. Para desenhar medidas mitigatórias de impacto ambiental dos empreendimentos em curso no nosso país (prestação de serviço que está no leque de atividades realizadas pelo MZUSP), é necessário conhecer a nossa biodiversidade. Gerar informação básica e fornecer material comparativo é parte vital de nossa missão. Os estudos visionários dos primeiros naturalistas em nosso país foram a base para a construção de uma longa tradição de pesquisa que nos coloca em uma posição-chave no cenário mundial. Uma série de políticas nacionais de incentivo à pesquisa em nossa área, promovidas pelo $\mathrm{CNPq}$, faz do Brasil um polo de estudos taxonômicos. Ainda assim, não temos o número de profissionais necessário para realizar os estudos de revisão de grupos importantes (Marques \& Lamas, 2006). A própria geração de conhecimento taxonômico requer muito tempo de análise comparativa de material por parte dos pesquisadores (Landim \& Hingst-Zaher, 2010). A esse quadro de déficit chamou-se de "impedimento taxonômico". 

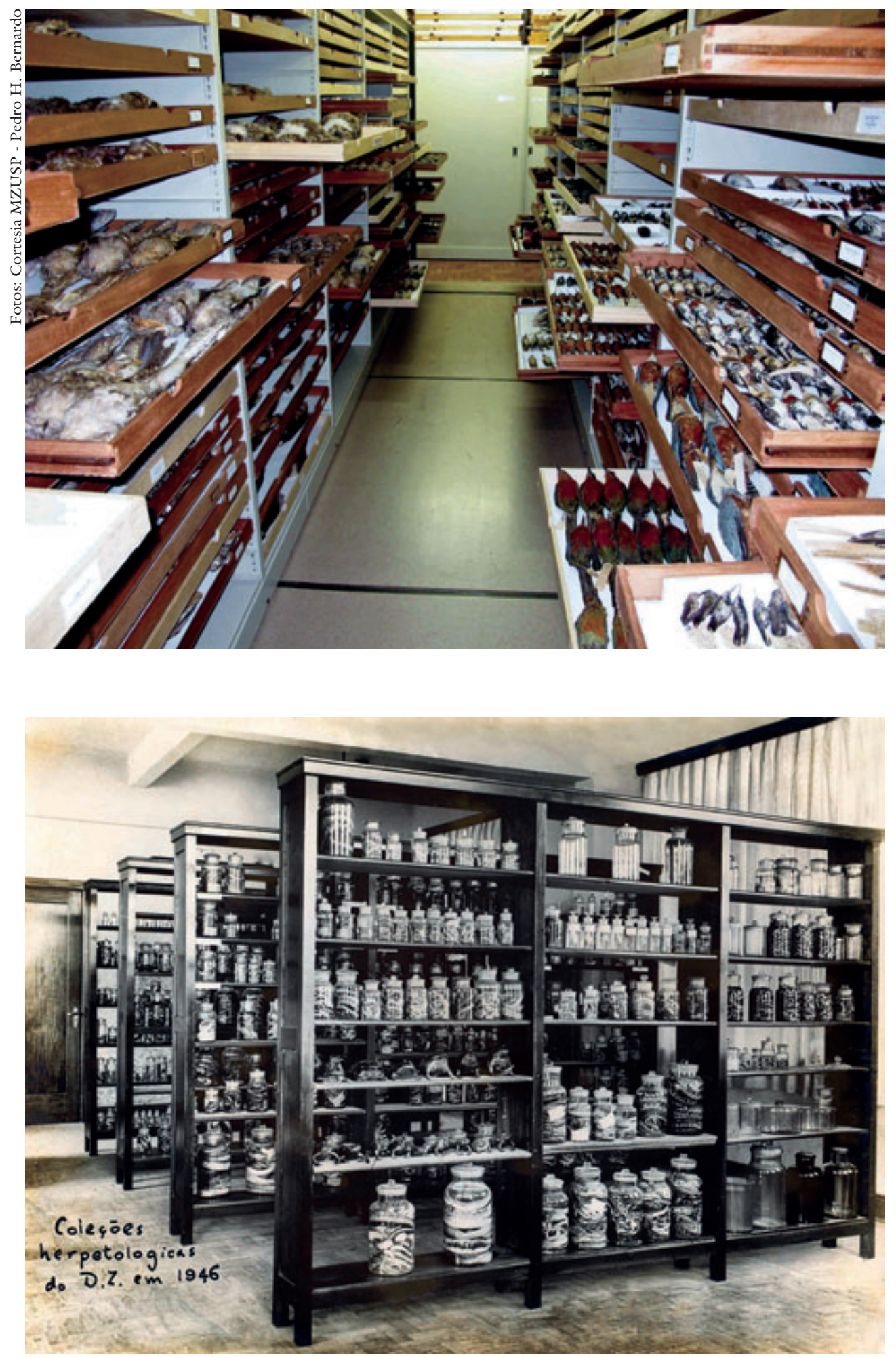

Figura 1 - (Abaixo) Coleção da Herpetologia do Departamento de Zoologia em 1946 (arquivos do MZUSP: autor desconhecido). (Acima) Coleção da Ornitologia do MZUSP em 2010. A maior coleção de aves brasileiras do mundo. 

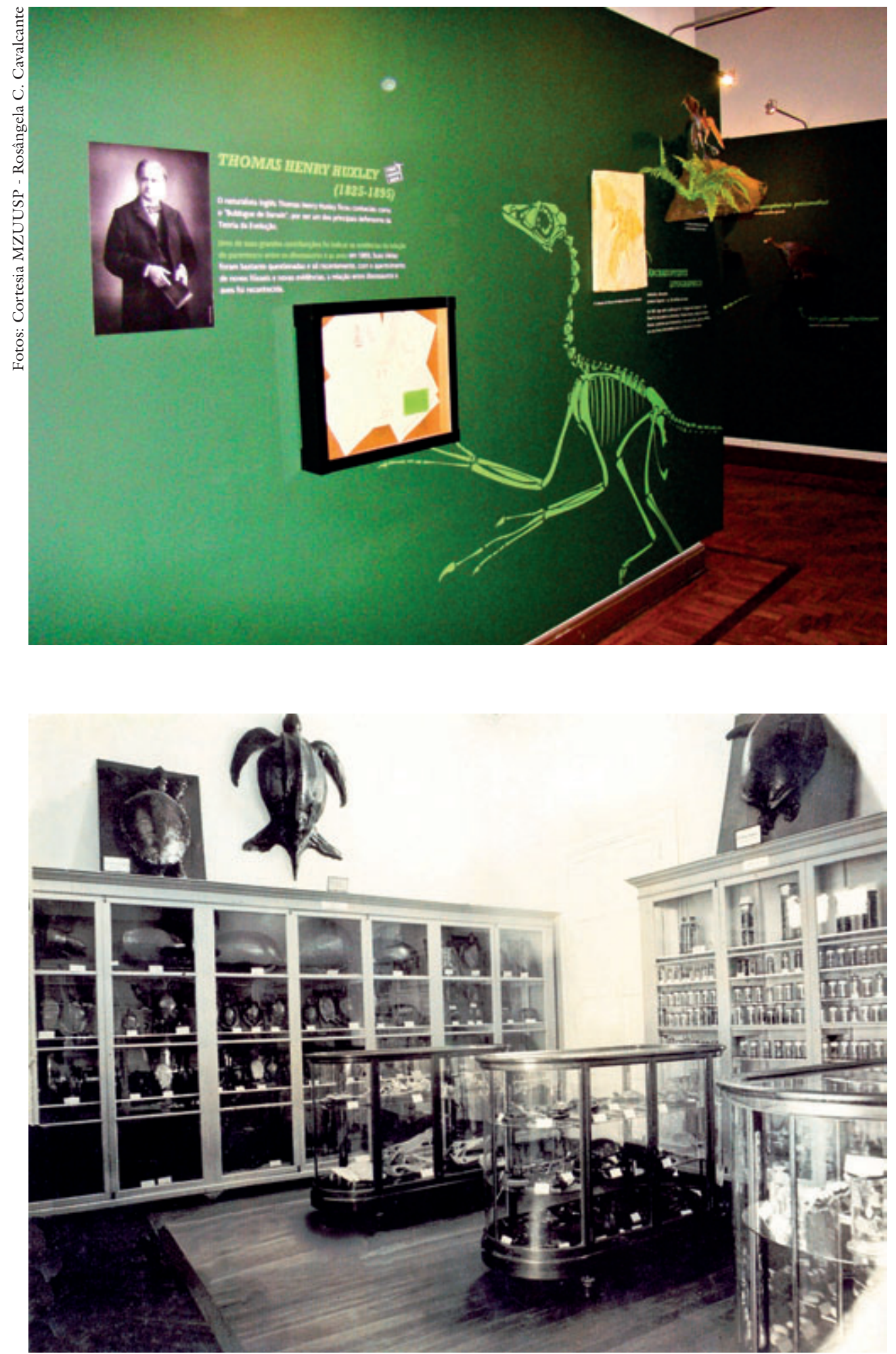

Figura 2 - (Abaixo) Exposição do Departamento de Zoologia na década de 1940 (arquivo do MZUSP: autor desconhecido). (Acima) Exposição temporária "Cabeça Dinossauro: o novo titã brasileiro", 2011. 
Desde 1954, o MZUSP é responsável pela administração da Estação Biológica de Boraceia, no município de Salesópolis, onde vem desenvolvendo atividades de pesquisa e ensino. As coletas estratégicas vinculadas a projetos de pesquisas financiados pela Fapesp e pelo $\mathrm{CNPq}$, assim como as geradas a partir de estudos de impacto ambiental iniciadas na era Vanzolini, viraram rotina na instituição e respondem ainda hoje pelo crescimento vertiginoso no número de exemplares mantidos no MZUSP.

Contemplados pelo edital de Infraestrutura da Fapesp, estamos em pleno processo de reestruturação dos sistemas de segurança e armazenagem de nossas coleções. O projeto contempla ainda a digitalização em uma base de dados comum (SophiAcervo) das coleções e disponibilização de informação sobre os exemplares tipo (exemplares de referência que devem ser analisados em estudos taxonômicos) na internet. Isso resultará em uma maior agilidade, tão almejada, em estudos em nossa área.

A exposição pública do MZUSP, porém, atravessou o século XX como que congelada no tempo e apenas pequenos esforços foram feitos no intuito de atualizá-la (Figura 2). Acompanhando o cenário museológico do final do século $\mathrm{XX}$ quando os museus assumiram seu papel social como meio de comunicação, $\mathrm{O}$ MZUSP iniciou um projeto novo de comunicação museológica. Isso se refletiu na reformulação de seu organograma com a criação de uma Divisão de Difusão Cultural (DDC) e dois serviços técnicos associados (Serviço de Atividades Educativas e Serviço de Museologia). Dessa forma, o MZUSP se distanciava da imagem de "Instituto de Pesquisa" construída a partir de meados do século XX. Foram contratados técnicos para os serviços da DDC e um docente para uma nova área de pesquisa em Museologia de Ciências e Divulgação Científica. ${ }^{3}$ Em 1998, o hall de exposição de longa duração entrou em reforma e foi reaberto em 2002 com uma nova exposição que privilegiava uma narrativa institucional à apresentação descontextualizada de animais taxidermizados. ${ }^{4}$ Em 2004, inauguramos uma galeria de exposições temporárias (Figura 2) que permitiu ao museu dinamizar seu projeto de comunicação. ${ }^{5}$ Essa estratégia possibilitou ao MZUSP promover a itinerância de suas exposições temporárias, o que começa a se transformar em rotina institucional. ${ }^{6}$ Em seus programas de comunicação, o MZUSP busca dialogar com seus visitantes sobre a Biodiversidade, mostrando como essa varia no tempo e no espaço. Sempre ressaltando a importância vital das coleções para gerar informação sobre o tema, o diálogo com o público articula problemas gerais das sociedades contemporâneas com a preservação patrimonial.

Em nosso país, os museus parecem viver a sua era entre meados do século XX e início do XXI. Com a criação da política dos museus, em 2003, o governo federal, por intermédio do Ministério da Cultura, inaugura uma série de políticas públicas de incentivo e regularização das atividades relacionadas aos museus. Esse movimento culmina com a lei do Estatuto dos museus de 2009 e sua influência pode ser sentida em diversas ações implementadas desde então, como 
a criação de diversos cursos de museologia em nosso país. Podemos atribuir a esse momento promissor aos museus brasileiros e à luta histórica por parte dos profissionais dos museus da USP a reforma no Estatuto da Universidade de São Paulo, no que concerne à administração dos seus museu estatutários.

O período de incorporação dos museus à USP, que nos traz aos dias atuais, não representou um período de calmaria e estabilidade institucional. Os museus universitários, em geral, sofreram com a falta de um nicho próprio nos estatutos das Universidades que possibilitasse o desenvolvimento pleno de suas atividades peculiares de estudo da cultura material e sua preservação e difusão, dentro e fora da Universidade. Um ponto crítico nesse processo foi a inserção dos pesquisadores dos museus na carreira de docente, com equiparação de direitos e salários (garantido no artigo 50 do Regimento Geral da USP de 1990) e o reconhecimento da curadoria como atividade didática. O outro foi a própria figura jurídica de Órgão de Integração atribuída aos museus desde 1988 e a criação de um organismo de tutela para eles na Universidade, a Coordenação de Museus. Com o fim dessa, na Resolução n.5.900 de dezembro de 2010 foi concedida aos museus a sua tão almejada maturidade administrativa. Os museus deixam de ser intermediados pela Coordenação dos Museus. Isso certamente dará aos museus da USP a agilidade administrativa de que eles precisam para lidar com tamanho crescimento, como o que pode ser observado no MZUSP. Neste momento, os regimentos e organogramas dos quatro museus estatutários estão sendo repensados para contemplar essa autonomia. Ficou ainda a questão da representatividade dos museus no Conselho Universitário. Mais do que nunca, isso nos parece ser apenas uma questão de tempo.

Como principais desafios do MZUSP, temos a falta de pessoal (ainda estamos longe da recomposição de nossos quadros no nível da antiga Secretaria de Agricultura que contava com 24 pesquisadores) e de espaço. Hoje, nossas coleções invadem cada milímetro de área livre que ainda pode ser encontrada no Museu. Além disso, uma área expositiva de cerca de 500 metros quadrados não faz jus ao nosso projeto de comunicação. Com o projeto Praça dos Museus, resgatado da década de 1990 e aprovado pela atual reitoria, essas questões serão sanadas. Nesse projeto, que implica a transferência do MZUSP para a Cidade Universitária, a área do MZUSP passaria dos atuais cerca de seis mil metros quadrados para cerca de 14 mil metros quadrados sem contar com a exposição. O projeto do arquiteto Paulo Mendes da Rocha contempla ainda a união da área expositiva do MZUSP à do Museu de Arqueologia e Etnologia (MAE) em um mesmo edifício (cilíndrico). Com sua inauguração prevista para 2013, o MZUSP e o MAE serão lançados em uma nova era dos museus da USP. Em uma nova configuração, reuniremos parte do acervo do antigo Museu Paulista (de História Natural). Ihering viveu a era da especialização, a nossa era é a da interdisciplinaridade. Também nos beneficiamos do novo status alcançado pelas disciplinas, inclusas na história natural, traduzidas no jargão atual como disci- 
plinas relacionadas ao estudo biodiversidade. Por essa razão, a prometida união entre MZUSP e MAE em uma exposição conjunta é uma oportunidade para trabalharmos temas transversais às ciências humanas e naturais.

Chegamos a 2011 com 14 docentes no MZUSP que respondem pela curadoria científica de suas coleções, pelo Programa de Pós-Graduação em Sistemática, Taxonomia Animal e Biodiversidade e pela colaboração na criação de um Programa de Pós-Graduação interunidades (ao lado dos demais museus estatutários da USP) em Museologia. Esse será o primeiro curso na área no Estado de São Paulo, atendendo a uma demanda crescente da atual política federal na área de museus. Em seu atendimento ao público, o número de visitantes do Museu vem aumentando a cada ano (ca. de 100 mil em 2010 quando implementamos o ingresso $2 \mathrm{em} \mathrm{l}$ em parceria com o Museu Paulista). Esse número será multiplicado com a itinerância sistemática das exposições. O Museu de Zoologia da USP inicia assim o século XXI atualizando o projeto inicial de Herman von Ihering, com inúmeras novas conquistas, mas sem se distanciar no essencial: excelência na pesquisa e na salvaguarda de seu acervo, no ensino e na comunicação com um público cada vez maior.

\section{Notas}

l Com cerca de 10 milhões de exemplares, nossa coleção é referência obrigatória para estudos de diversos grupos de animais.

2 O Brasil possui cerca de $20 \%$ de toda a biodiversidade do planeta.

3 Hoje a DDC do MZUSP conta com uma docente, dois técnicos (nível superior e médio) no Serviço de Museologia e um técnico de nível superior no Serviço Educativo.

4 A exposição de longa duração que desde 2002 é apresentada ao público sofrendo pequenas alterações é "Pesquisa em Zoologia: a biodiversidade sob o olhar do zoólogo".

5 De 2004 a 2011, 11 exposições temporárias foram exibidas no MZ (as três últimas foram: "Crise da biodiversidade: a natureza ameaçada", "Charles Darwin: evolução para todos!" e "Cabeça Dinossauro: o novo titã brasileiro").

6 A exposição "Moluscos joias do mar" se tornou itinerante (será exibida no Museu Ciência e Vida de Duque de Caxias, RJ, a partir de julho) e outras duas, "Charles Darwin: evolução para todos!" e "Cabeça Dinossauro: o novo titã brasileiro", se preparam para itinerância.

Referências

ALVES, A. M. A. O Ipiranga apropriado: ciência, política e poder. O Museu Paulista, 1893-1922. São Paulo: Humanitas, 2001. 
BRANDÃO, C. R.; COSTA, C. Uma crônica da integração dos museus estatutários à USP. Anais do Museu Paulista. v.15, p.207-17, 2007.

BRANDÃO, C. R.; LANDIM, M. I. Para que servem os museus? São Paulo: Secretaria de Estado da Cultura SP (no prelo). (Col. "Museu Aberto", v.2).

BRANDÃO, C. R.; SAMARA, E. M. Da difusão à pesquisa no Estado de São Paulo. A história do Museu Paulista da Universidade de São Paulo. Revista de Cultura e Extensão da USP, n.0, p.94-103, 2005.

LANDIM, M. I.; HINGST-ZAHER, E. Brazil's Biodiversity Crisis. ICOM News, v.63, n.2, p.14-5, Oct. 2010.

LOPES, M. M. O Brasil descobre a pesquisa científica: os museus e as ciências naturais no século XIX. São Paulo: Hucitec, 1997.

LOPES, M. M.; FIGUEIRÔA, S. F. A criação do Museu Paulista na correspondência de Hermann von Ihering (1850-1930). São Paulo: Anais do Museu Paulista. v.10/11, p.23-35, 2002-2003.

MARQUES, A. C.; LAMAS, C. J. E. Taxonomia zoológica no Brasil: o estado da arte, expectativas e sugestões de ação futura. Papéis Avulsos de Zoologia. v.46, n.13, p.139-74. 2006.

MENEZES, N. A. et al. Ichthyological Collection Building at the Museu de Zoologia da Universidade de São Paulo. In: PIETSCH, T. W.; ANDERSON JUNIOR, W. D. (Org.) Collection Building in Ichthyology and Herpetology. The American Society of Ichthyologists and Herpetologists. Special publication n.3, p.561-65, 1997.

NEVES NETO, C. S. das. Christiano Stokler das Neves, o arquiteto concreto. São Paulo: Dialeto. 2008.

SHEETS-PYENSON, S. Cathedrals of Science: the development of colonial natural history museums during the late nineteenth century. Kingston: McGill-Queen's University Press, 1988.

SILVA, M. C. Christiano Stockler das Neves e o Museu de Zoologia da Universidade de São Paulo. São Paulo, 2006. 274p. Dissertação (Mestrado em Arquitetura) - Faculdade de Arquitetura e Urbanismo, Universidade de São Paulo.

STEARN, W. The National History Museum at South Kensington. London: Natural History Museum, 1998.

YANNI, C. Nature's museums: Victorian science and the architecture of display. New York: Princeton Architectural Press, 2005.

RESUMO - Museus ditos tradicionais têm como centro de gravidade seus acervos. Estes, muitas vezes, precedem as próprias instituições: o clássico modelo de coleções privadas que encontram as vias institucionais públicas e a acessibilidade. O Museu de Zoologia da Universidade de São Paulo (MZUSP) não é uma exceção a essa regra. Seu acervo está vinculado a coleções do século XIX, incorporadas ao Estado de São Paulo, em idas e vindas institucionais, até a configuração atual de museu universitário de excelência em pesquisa, ensino e extensão. Como tal, o MZUSP continua enfrentando desafios e necessidades de transformação e adaptação às novas realidades do século XXI.

PALAVRAS-CHAVE: Museus de História Natural, Museus Universitários, Biodiversidade. 
ABSTRACT - "Traditional" museums have their center of gravity in collections that often precede the institutions themselves. It is the classic model of private collections that meet the public institutional channels and accessibility. The Museum of Zoology of the University of São Paulo is not an exception to this rule. Its collection is linked to collections of the nineteenth century incorporated to the State of São Paulo, in comings and goings of institutional settings to the current status as university museum of excellence in research, teaching and outreach. As such, MZUSP continues to face challenges and changing needs and adapt to new realities of the twenty-first century.

KEYWORDS: Natural History Museums, University Museums, Biodiversity.

Maria Isabel Landim é professora da Divisão de Difusão Cultural do Museu de Zoologia da USP (desde dezembro de 2008); Board (2011-2013) International Comittee for Museum and Collections of Natural History from International Council of Museums (ICOM Nathist). @ - milandim@usp.br

Recebido em 15.8.2011 e aceito em 19.8.2011. 